\title{
Erratum to: Selective Embolization of Systemic Collaterals \\ for the Treatment of Recurrent Hemoptysis Secondary to Unilateral Absence of Pulmonary Artery in a Child
}

\author{
Yin $\mathrm{Zhou}^{1} \cdot$ Jiaywei $\mathrm{Tsauo}^{2} \cdot \mathrm{Yuan}_{\mathrm{Li}^{1}} \cdot \mathrm{Xiao}_{\mathrm{Li}^{2}}$
}

Published online: 25 November 2015

(C) Springer Science+Business Media New York and the Cardiovascular and Interventional Radiological Society of Europe (CIRSE) 2015

\section{Erratum to: Cardiovasc Intervent Radiol (2015) 38:1312-1315 DOI: 10.1007/s00270-014-0965-3}

A section of the article text was mistakenly removed from the published text. In the Case section of the article, a section of the paragraph currently reads:

The pulmonary artery pressure was normal within limits. Thoracic descending aortography confirmed the presence of bronchial systemic collaterals in the left lung (Fig. 2B). The patient recovered uneventfully and was discharged on the following day. She remained asymptomatic with no recurrence of hemoptysis at 8 months follow-up.

The section should say:

The pulmonary artery pressure was normal within limits. Thoracic descending aortography confirmed the presence of bronchial systemic collaterals in the left lung (Fig. 2B).
Selective bronchial angiography performed with a 4-French Cobra 2 catheter (Terumo, Tokyo, Japan) showed dilated, tortuous systemic collaterals supplying the entire left lung with parenchymal staining (Fig. 2C). Selective embolization was performed by coaxially placing a 2.5-French microcatheter (Mira-Flex; Cook, Bloomington, IN, USA) into the branches of the bronchial artery and using 2-3-mm diameter microcoils (Tornado; Cook) (Fig. 2D). The procedure time was $42 \mathrm{~min}$, the fluoroscopy time was $11 \mathrm{~min}$, and the dose area product (DAP) was $13,487 \mathrm{mGy} \mathrm{cm}^{2}$. The patient recovered uneventfully and was discharged on the following day. She remained asymptomatic with no recurrence of hemoptysis at 8 months follow-up.

The online version of the original article can be found under doi:10.1007/s00270-014-0965-3.

Xiao Li

simonlixiao@gmail.com

Yin Zhou

zhouyin502@163.com

Jiaywei Tsauo

80732059@qq.com

Yuan Li

liyuanletters@163.com

1 Department of Pediatric Surgery, West China Hospital, Sichuan University, Chengdu 610041, Sichuan, China

2 Institute of Interventional Radiology, West China Hospital, Sichuan University, Chengdu 610041, Sichuan, China 